profiling was carried out using Illumina chip array technology. After mapping genome-wide methylation quantitative trait loci (mQTLs) in cis $(<1 \mathrm{Mb})$, we focused on known RA risk loci, integrating paired normalised gene expression measurements for transcripts within $500 \mathrm{~kb}$ of index CpGs. We also sought trans mQTLs, highlighting RA-specific effects.

Results CD4+ T lymphocyte cis-mQTLs co-localised with 30 independent $(\mathrm{r} 2<0.8)$ RA-associated SNPs, whilst in B lymphocytes such mQTL effects were present at 31 RA SNPs. 80\% of these variants functioned as cis-mQTLs in both cell types. CpG sites subject to cis effects at risk loci were depleted in regions associated with cell type-specific repressed chromatin marks, with enrichment at enhancer regions and those flanking transcription start sites, suggesting active roles in transcriptional regulation. Linear regression identified regulatory effects of these $\mathrm{CpG}$ sites on gene expression, and causal inference testing highlighted genes for which risk SNPs most likely modulate gene expression via $\mathrm{CpG}$ methylation. Such effects, robust to false discovery rate, were particularly prevalent in CD4+ $\mathrm{T}$ lymphocytes, for example implicating ANKRD55, ORMDL3, and FCRL3 as causal genes in this cell type. Our analysis of mQTLs acting in trans identified inter-chromosomal SNP-CpG associations, also revealing instances of differential effect size in RA patients and controls.

Conclusions Here we highlight an important mechanism by which genetic variants may contribute to altered lymphocyte phenotype, and demonstrate the utility of DNA methylation profiling as a tool for the prioritization of candidate genes following GWAS studies in RA. The functional roles of highlighted genes in CD4 $+\mathrm{T}$ cells during RA pathogenesis await clarification.

Disclosure of Interest None declared.

\section{P107 POLYMORPHISMS IN SLC2A9 AND SLC22A12 GENES ARE RELATED TO HYPERURICEMIA, GOUT AND ALSO TO HYPOURICEMIA}

\footnotetext{
1,2K Pavelcova*, 1,2J Bohata, ${ }^{1} \mathrm{~K}$ Pavelka, 1,3B Stiburkova. 'Institute of Rheumatology; ${ }^{2}$ Department of Rheumatology, First Faculty of Medicine, Charles University; ${ }^{3}$ Department of Pediatrics and Adolescent Medicine, First Faculty of Medicine, Charles University and General University Hospital in Prague, Prague, Czech Republic
}

10.1136/annrheumdis-2018-EWRR2019.95

Career situation of first and presenting author Student for a master or a $\mathrm{PhD}$.

Introduction Serum uric acid concentration is significantly influenced by urate transporters, such as ABCG2 (encoded by ABCG2 gene), GLUT9 (SLC2A9 gene) and URAT1 (SLC22A12 gene). The main function of ABCG2 is uric acid secretion, whereas GLU9 and URAT1 also ensure reabsorption. Pathogenic allelic variants in SLC2A9 and SLC22A12 are not only associated with hyperuricemia and gout, but they also lead to rare hereditary renal hypouricemia (type 1 - OMIM \#220150 or type 2 - OMIM \# 612076).

Objectives Previously, we analyzed $A B C G 2$ gene and detected non-synonymous variants that lead to hyperuricemia and early onset of the gout. ${ }^{1}$ The aim of this study was to find a possible correlation between variants in SLC2A9 and SLC22A12 and hypouricemia, hyperuricemia and gout. ${ }^{2}$

Methods We recruited a cohort of 232 individuals with primary gout and hyperuricemia. We examined coding regions of
SLC2A9 (13 exons) and SLC22A12 (10 exons) by Sanger sequencing. We also analyzed SLC2A9 and SLC22A12 in five patients with suspect hypouricemia.

Results In the cohort of 232 individuals, we detected five synonymous variants, 18 intron variants and seven missense variants in SLC2A9: A17T, G25R, T275M, D281H, V282I, $\mathrm{R} 294 \mathrm{H}$, and P350L. In SLC22A12 gene, we found six synonymous variants and seven intron variants.

We detected several pathogenic variants in patients with suspect hypouricemia. Intronic variant c.1419+1G $>A$ in SLC2A9 most likely affects the splicing. In SLC22A12, we found rare pathogenic variants T467M and L415_G417del. These variants have according to our previous study high frequency in the Czech and Slovak Roma population. ${ }^{3}$

Conclusions The uric acid level is determined by a complex mechanism that is not yet fully understood. Disorders of urate transporters can not only lead to hyperuricemia, but in rare cases also to hypouricemia.

\section{REFERENCES}

1. Stiburkova B, et al. Rheumatology (Oxford) 2017 November 1:56(11):19821992. doi:10.1093/rheumatology/kex295

2. Hurba O, et al. PLoS One 2014 September 30;9(9):e107902. doi:10.1371/journal. pone. 0107902

3. Gabrikova D, et al. Urolithiasis 2015 October;43(5):441-5. doi:10.1007/s00240015-0790-4

Acknowledgements This study was supported by the grant from the Czech Republic Ministry of Health AZV 15-26693A. Disclosure of Interest None declared.

\section{P108/028 FATTY ACID OXIDATION CAN DRIVE HUMAN MONOCYTE DERIVED CCL2O IN THE RA SYNOVIAL ENVIRONMENT}

\begin{abstract}
${ }^{1,2} \mathrm{LC}$ Rodgers ${ }^{*},{ }^{2} \mathrm{~J}$ Cole, ${ }^{3} \mathrm{~K}$ Rattigan, ${ }^{3,4} \mathrm{MP}$ Barrett, ${ }^{5} \mathrm{~N}$ Kurian, ${ }^{1} \mathrm{IB}$ Mclnnes, ${ }^{1,2} \mathrm{CS}$ Goodyear. ${ }^{1}$ Centre of Immunobiology, Institute of Infection, Immunity and Inflammation, College of MVLS; ${ }^{2}$ GLAZgo Discovery Centre, Institute of Infection, Immunity and Inflammation, College of MVLS; ${ }^{3}$ Wellcome Centre for Molecular Parasitology, Institute of Infection, Immunity and Inflammation, College of MVLS; ${ }^{4}$ Glasgow Polyomics, Wolfson Wohl Cancer Research Centre, College of MVLS, University of Glasgow, Glasgow, UK; ${ }^{5}$ Precision Medicine and Genomics, RIA Precision Medicine Unit, IMED Biotech Unit, AstraZeneza, Gothenburg, Sweden
\end{abstract}

10.1136/annrheumdis-2018-EWRR2019.96

Career situation of first and presenting author Post-doctoral fellow.

Introduction Metabolic pathways are considered to have a governing role in inflammatory cascades in myeloid cells. This is particularly evident in murine macrophages where glycolysis and fatty acid oxidation (FAO) have been implicated in inflammatory cascades and immune regulation respectively. ${ }^{1}$ However, investigation of intracellular metabolism of human monocytes in the context of the hypoxic and inflammatory RA synovium is lacking.

Objectives To mimic the hypoxic RA environment in vitro and metabolically profile human monocytes. Determine if altered metabolic pathways have a functional impact on monocytes under disease-relevant conditions.

Methods Human monocytes were isolated from buffy coats and were exposed to hypoxia in vitro. Metabolic profiling of monocytes was carried out by LC-MS metabolomics. Inflammatory mediator release after LPS or RA-synovial fluid (RA- 
SF) stimulation was analysed by ELISA. FAO was inhibited by etomoxir or enhanced with exogenous carnitine supplements. Transcriptomics of RA blood monocytes and RA-SF macrophages was carried out by microarray.

Results We report that hypoxia exacerbates CCL20 and IL$1 \beta$ release in response to LPS and increases glycolytic intermediates at the expense of carnitines. Modulation of carnitine identified a novel role for FAO in the production of CCL20 in response to LPS. Transcriptomics of RA blood monocytes and RA-SF macrophages revealed that fatty acid metabolism was altered and CCL20 was increased when monocytes enter the RA milieu. In vitro analysis of monocytes showed that RA-SF increases carnitine abundance and CCL20 production in hypoxia, which was exacerbated by exogenous carnitine.

Conclusions This work has revealed a novel inflammatory mechanism in RA which links FAO to CCL20 production in human monocytes. This may contribute to RA disease pathogenesis by promoting the recruitment of Th17 cells and osteoclastogenesis. ${ }^{2} 3$

\section{REFERENCES}

1. O'Neill LA, et al. Nat Rev Immunol 2016;16:553-565.

2. K Hirota, et al. J Exp Med 2007;204:2803-2812.

3. G Lisignoli, et al. J Cell Physiol 2009;221:154-160.

Disclosure of Interest None declared.

\section{P109 EXPRESSION LEVELS OF MIR-21 AND MIR-29 IN THE SERUM OF SYSTEMIC SCLEROSIS PATIENTS}

${ }^{1} \mathrm{R}$ Shumnalieva*, ${ }^{2} \mathrm{D}$ Kachakova, ${ }^{3} \mathrm{~V}$ Shoumnalieva-Ivanova, ${ }^{4} \mathrm{P}$ Miteva, ${ }^{2} \mathrm{R}$ Kaneva, ${ }^{1} \mathrm{Z}$ Kolarov, ${ }^{1} \mathrm{~S}$ Monov. ${ }^{1}$ Department of Internal Medicine, Clinic of rheumatology; ${ }^{2}$ Molecular Medicine Center, Department of Medical Chemistry and Biochemistry, Medical University - Sofia; ${ }^{3}$ IQvia-Bulgaria; ${ }^{4}$ University Hospital 'Prof. Ivan Mitev', Medical University - Sofia, Sofia, Bulgaria

10.1136/annrheumdis-2018-EWRR2019.97

Career situation of first and presenting author Assistant.

Introduction Recent studies reveal the important role of microRNAs (miRNAs) in the pathogenesis of systemic sclerosis (SSc). miRNAs are involved in key biological pathways that regulate the fibrotic process in SSc. ${ }^{1}$ Deregulated expression of miRNAs has been found in the serum, in skin tissues, in skin fibroblasts as well as in endothelial cells of patients with $\mathrm{SSc}^{2}$

Objectives The aim of our study was to evaluate the expression levels of miR-21 and miR-29 in the serum of SSc patients and to determine their correlation with clinical and immunological parameters.

Methods 34 patients fulfilling the ACR/EULAR 2013 classification criteria for SSc were included in the study. miR-21 and miR-29 expression levels in the serum were determined by PCR (SYBR Green technology). $2^{-\Delta \Delta C t}$ method was used for analysis. 14 healthy donors were used as controls.

Results Expression levels of miR-21 were upregulated in the serum of $17(50.0 \%)$ of the patients. The expression of miR29 was downregulated in $15(44.12 \%)$ of the patients. Receiver operating characteristic (ROC) curve analysis was conducted in order to evaluate the diagnostic accuracy of the expression levels of the studied miRNA in the serum. Area under the curve (AUC) for miR-21 was $0.634 \quad(95 \%$ $\mathrm{CI}=0.479-0.790), \mathrm{p}=0.147$ with $64.7 \%$ sensitivity and $64.3 \%$ specificity. AUC for miR-29 was 0.605 (95\% CI $=0.420$ 0.790 ), with $64.3 \%$ sensitivity and $52.9 \%$ specificity but without statistical significance $(p=0.257)$. The multimarker analysis of the ROC curves for both miRNAs showed $\mathrm{AUC}=0.714$ (95\% CI $=0.569-0.860), \mathrm{p}=0.021$ with $79.4 \%$ sensitivity and $42.9 \%$ specificity. Levels of miR-29 correlated with the levels of miR-21 in the serum (with Spearman correlation coefficient $0.517, p=0.00017)$ and with the presence of anti-Scl70 antibodies in the serum (with Spearman correlation coefficient $0.438, \mathrm{p}=0.010$ ).

Conclusions Our data showed a deregulation of miR-21 and miR-29 in the serum of patients with SSc which could suggests their potential role in the disease pathogenesis. Further analysis with higher number of patients is needed to confirm if these miRNAs could be used in the clinical practice as diagnostic biomarkers as well as biomarkers for both disease activity and progression.

\section{REFERENCES}

1. Li Y, Huang J, Guo M, Zuo X. MicroRnAs Regulating Signaling Pathways: Potential Biomarkers in Systemic Sclerosis. Genomics, Proteomics \& Bioinformatics. 2015;13(4):234-241.

2. Maurer B, Stanczyk J, Jüngel $A$, et al. MicroRNA-29, a key regulator of collagen expression in systemic sclerosis. Arthritis \& Rheumatism 2010;62:17331743.

Acknowledgements The study was supported by Grant 62/ 2015 funded by Medical University - Sofia, Bulgaria.

Disclosure of Interest None declared.

\section{P110/018 EPIGENETIC CHANGES BY INHIBITION OF DOT1L AFFECT WNT SIGNALING, PROLIFERATION AND CELL CYCLE IN DERMAL FIBROBLASTS, WITH NO OVERALL EFFECT ON COLLAGEN DEPOSITION IN MODELS OF FIBROSIS}

${ }^{1,2} \mathrm{~N}$ Berghen* ${ }^{3}{ }^{3} \mathrm{C}$ Cremer, ${ }^{1,2} \mathrm{E}$ De Langhe, ${ }^{1,2} \mathrm{R}$ Lories. ${ }^{1}$ Department of Development and Regeneration, KU Leuven; ${ }^{2}$ Division of Rheumatology, UZ Leuven; ${ }^{3}$ Translational Research in Gastrolntestinal Disorders, KU Leuven, Leuven, Belgium

\subsection{6/annrheumdis-2018-EWRR2019.98}

Career situation of first and presenting author Student for a master or a $\mathrm{PhD}$.

Introduction The role of epigenetic factors in the pathophysiology of fibrosis, a hallmark of Systemic Sclerosis, is increasingly explored. DOT1L, the unique H3K79. methyltransferase, methylates histone 3 at the Lysine residue at position 79 , thereby regulating gene expression programs. In cartilage and bone, DOT1L has cell-type specific effects on Wnt signaling, a pathway suggested to play an important role in fibrosis.

Objectives To study the role of DOT1L in fibrosis.

Methods Primary human dermal fibroblasts were treated with DOT1L-inhibitor EPZ-5676 or vehicle and stimulated with TGF- $\beta$. Expression of smooth muscle alpha 2 actin (ACTA2) and Wnt target genes was measured by RT-qPCR. Western Blot was done for dimethylated H3K79 and $\beta$-catenin. Picrosirius Red staining measured collagen deposition. 5-Bromo-2'-deoxy-uridine (BrdU) labeling for proliferation and flow cytometry with Propidium Iodide for cell cycle analysis was done. Col1a2;Cre-ERT ${ }^{2}$;DOT1 $11^{\mathrm{fl} / \mathrm{fl}}$ mice, injected 